\title{
A review of nondestructive examination technology for polyethylene pipe in nuclear power plant
}

(C) The Author(s) 2018. This article is published with open access at link.springer.com and journal.hep.com.cn

\begin{abstract}
Polyethylene (PE) pipe, particularly highdensity polyethylene (HDPE) pipe, has been successfully utilized to transport cooling water for both non-safety- and safety-related applications in nuclear power plant (NPP). Though ASME Code Case N755, which is the first code case related to NPP HDPE pipe, requires a thorough nondestructive examination (NDE) of HDPE joints. However, no executable regulations presently exist because of the lack of a feasible NDE technique for HDPE pipe in NPP. This work presents a review of current developments in NDE technology for both HDPE pipe in NPP with a diameter of less than $400 \mathrm{~mm}$ and that of a larger size. For the former category, phased array ultrasonic technique is proven effective for inspecting typical defects in HDPE pipe, and is thus used in Chinese national standards GB/T 29460 and GB/T 29461. A defectrecognition technique is developed based on pattern recognition, and a safety assessment principle is summarized from the database of destructive testing. On the other hand, recent research and practical studies reveal that in current ultrasonic-inspection technology, the absence of effective ultrasonic inspection for large size was lack of consideration of the viscoelasticity effect of PE on acoustic wave propagation in current ultrasonic inspection technology. Furthermore, main technical problems were analyzed in the paper to achieve an effective ultrasonic test method in accordance to the safety and efficiency requirements of
\end{abstract}

Received September 25, 2017; accepted December 19, 2017

Jinyang ZHENG, Yue ZHANG, Dongsheng HOU, Yinkang QIN, Jianfeng SHI (凹)

Institute of Process Equipment, Zhejiang University, Hangzhou 310027, China

E-mail: shijianfeng@zju.edu.cn

Weican GUO

Zhejiang Provincial Special Equipment Inspection and Research Institute, Hangzhou 310027, China

Chuck ZHANG

Georgia Tech Manufacturing Institute, Georgia Institute of Technology, Atlanta, GA 30332, USA related regulations and standards. Finally, the development trend and challenges of NDE test technology for HDPE in NPP are discussed.

Keywords polyethylene pipe, nuclear power plant, ultrasonic inspection, nondestructive testing, safety assessment

\section{Introduction}

Most of Chinese mainland's electricity is produced from fossil fuels, predominantly coal. Rapid economic growth has led to power shortages, and the reliance on fossil fuels has increasingly resulted in more serious air pollution. Nuclear power in China is now under vigorous development due to its excellent advantages of high input-output energy ratio, low pollution emission, and long operation life [1].

Metallic piping, such as carbon steel piping, is historically the most commonly used piping for pressurized cooling water transportation. However, metallic piping is prone to corrosion, fouling, and microbiological attack, therefore resulting in greater costs for frequent maintenance (2-3 years) or replacement (about 10 years) and potential safety hazards [2]. High density polyethylene (HDPE) pipe is a new option for nuclear power plant (NPP) worldwide. In 1995, HDPE was first applied in a non-safety service water system of Catawba nuclear station in the US by Duke Energy, and has been effectively in service without corrosion or fouling problems for over 20 years [3]. Due to its significant advantages such as corrosion resistance, strength-to-weight ratio, flexibility, and long service life (50 years) [4], HDPE used in water piping at power plants has been proven to be efficient and cost effective. In 2009, the US Nuclear Regulatory Commission (NRC) issued Catawba safety evaluation. Afterwards, HDPE piping could be used for Class 3 safety related piping with NRC approval, such as the cooling water lines in Callaway Nuclear Plant [5] and Catawba Nuclear Plant Unit 2. Currently, HDPE piping has been 
utilized in the latest third generation nuclear power reactors, such as AP1000 designed by American Westinghouse Company and Hualong One designed by China National Nuclear Corporation and China General Nuclear Power Group.

The nuclear industry in the US is drafting ASME Code Case N-755 that contains the requirements for NPPs applications for HDPE pipe [6]. N-755 requires that visual examination, hydrostatic test, and nondestructive examination (NDE) shall be conducted on all pipe fusion joints before operation [7]. Visual examination includes inspection of the general surface for indentations, review and verification of fusion data for the joint, and evidence of leakage during the hydrostatic test. Hydrostatic test requires that no leakage shall occur during the process of pressurization, pressure maintenance, and pressure reduction. However, only obvious surface damages can be found through visual examination and the inspection effectiveness is largely depended on personnel qualification. Hydrostatic tests are generally effective if gross throughwall flaws exist in the fusion joint [8]. For detecting fusion flaws with potential detriments to operation safety, volumetric examination should be performed, and results should be evaluated by qualified NDE personnel. Though ASME has approved Code Case N-755, the NRC has not endorsed N-755. One of the major reasons is the lack of practical NDE procedures and qualification [9]. N-755 contains rules for personnel qualification requirements, but it does not specify NDE method, NDE procedures, or acceptance criteria.

Currently, no NDE technology has proved reliable with high sensitivity and resolution for the inspection needs from the nuclear industry. China has approved and issued two national standards $[10,11]$ on the NDE and safety assessment of electrofusion (EF) joints of PE pipes with thickness of $40-400 \mathrm{~mm}$ for natural gas transportation. The standards include specific ultrasonic inspection procedures and qualifications. Nevertheless, the existing ultrasonic testing technology encountered new challenges and obstacles when it was applied for inspecting PE pipe joint of a larger diameter in NPP. When ultrasonic wave propagates in PE, wave distortion will occur because of sound attenuation and sound dispersion. The former makes sound energy decrease with the transmission distance, and the latter can distort the pulse wave due to the different phase velocity of the different frequency components of the sound signal. The distortion will gradually accumulate with the increase of the propagation distance which will affect the focusing and imaging of sound waves. The conventional ultrasonic testing system assumes that the material to be tested is linear elasticity and uses ideal wave equation (constant sound velocity) as the theoretical basis to design the delay time and to realize focusing and imaging. However, for large size PE pipe whose material is viscoelastic, sound attenuation and frequency dispersion effects will accumulate with the propagation distance and will be heavily distorted, so the ideal wave equation will not be able to accurately describe the actual propagation law. Therefore, establishment of a phased array sound field model suitable for PE and accurate prediction of sound pressure distribution during long distance propagation are important prerequisites for developing nuclear PE ultrasonic detection technology.

This paper systematically reviewed current ultrasonic inspection techniques for PE pipe and their applications. Situation and progress of study on technical obstacles of ultrasonic testing for PE pipe in NPP were then presented. Finally, prospects of NDE technology development for PE pipe in NPP were further discussed.

\section{Current ultrasonic inspection techniques for PE pipe}

Defects in EF joints of PE pipes can be categorized to poor fusion interface, void, structural deformity (dislocation of heating wires), and cold welding [12]. Meanwhile, defects in butt fusion (BF) joints are classified as crack, poor fusion interface, void, and cold welding [13]. Cold welding is caused by improper welding power or welding time. Such PE joint can survive in normal hydraulic testing for qualification but could still cause leakage failure in the operation [14]. Cold welding defect is correlated with the eigen-line, which is formed due to a polymer chain orientation in the welding region of PE joint [15]. As there is no macro change in a cold welding joint structure, most NDE methods are invalid for detecting cold welding defect. However, an effective NDE method for PE joints should be reliable to detect all typical defects of required sizes, and cold welding is the main obstacle for the development of NDE technique for PE joints.

\subsection{Nondestructive examination methods for PE pipe}

X-ray technique, infrared thermal imaging technique and ultrasonic inspection technique are currently used NDE methods for PE pipe. X-ray technique has been widely applied in assessing metal welding quality [16,17], but can only detect volumetric defects (void or inclusion) in PE joints with low X-ray absorption capability. Infrared thermal imaging technology has a deficiency of low defect resolution with a minimum detectable diameter of $8 \mathrm{~mm}$ at a depth of $10 \mathrm{~mm}$ [18]. Ultrasound technique was applied to detect voids, inclusions, and cracks at the fusion region in BF joints, and the defect sizes detected were 5,10 , and $12 \mathrm{~mm}$ for BF joints, respectively (DN160, SDR11) [19]. Therefore, traditional ultrasonic inspection technique was proven effective in detecting most typical defects in BF joints. However, the inspecting resolution needs to be enhanced.

Recently, the Welding Institute (TWI) from the UK applied time of flight diffraction (TOFD) ultrasonic testing 
technique to inspect $\mathrm{BF}$ joints of PE pipe (less than 400 $\mathrm{mm}$ in diameter) [20]. Compared with traditional ultrasound testing method, this technique achieved excellent inspecting resolution, but failed in detecting cold welding defect. Zhejiang University applied phased array ultrasonic technique (PAUT) and managed to inspect all typical flaws in both EF joint [21] and BF joint [13]. For instance, the welding regions in EF joint of D200 and BF joint of D200 are clearly shown in Figs. 1 and 2, and the detected voids have a width of 2 and $4 \mathrm{~mm}$, respectively. PAUT has

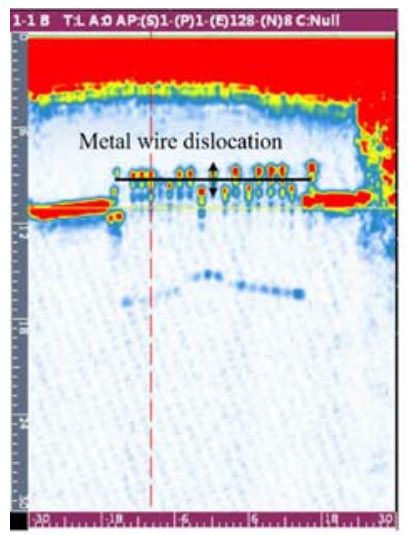

(a)

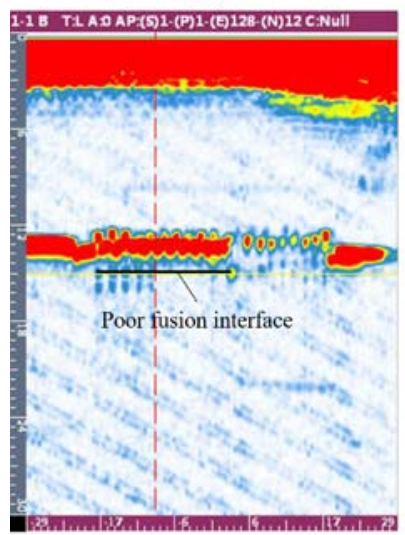

(c)

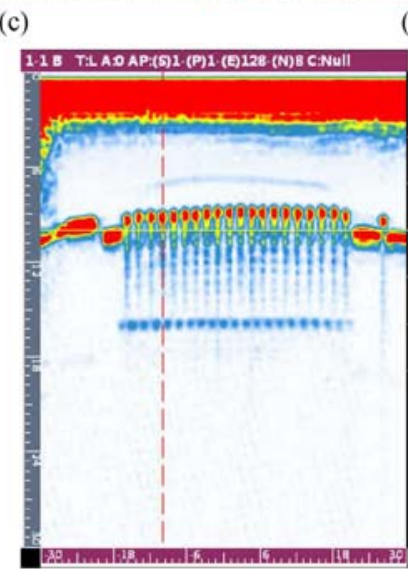

(e)

Fig. 1 Typical ultrasonic image of all types of defects in EF joint of PE pipe (less than DN200) [21]. (a) Metal wire dislocation; (b) void; (c) poor fusion interface; (d) cold welding; (e) normal
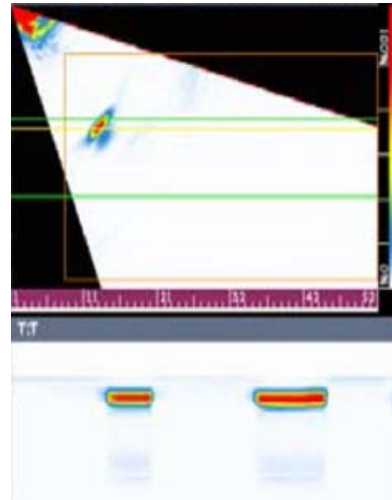

(a)

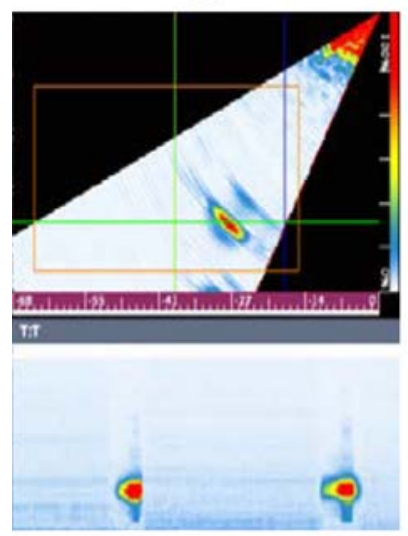

(c)

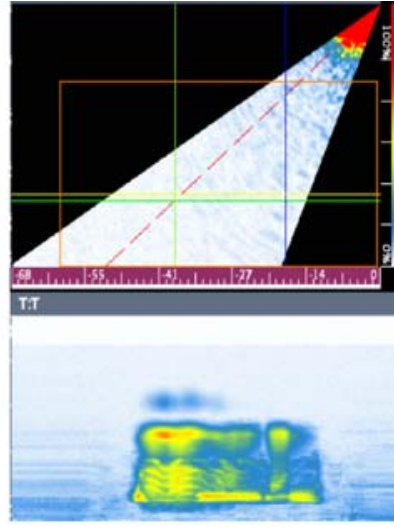

(b)

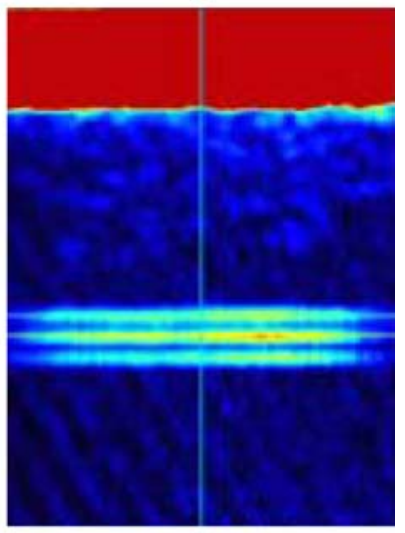

(d)

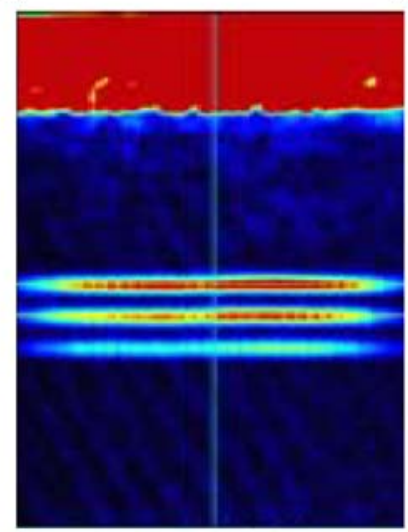

(e)

Fig. 2 Typical ultrasonic image of all types of defects in BF joint of PE pipe (less than DN200) [13]. (a) Crack; (b) poor fusion interface; (c) void; (d) cold welding; (e) normal

emerged as a rapid NDE technique for the detection and imaging of defects in PE joints due to its flexibility in varying the focusing of beam to a point of interest.

\subsection{Phased array ultrasonic inspection technology}

Ultrasonic inspection system based on phased array technique normally consists of phased-array ultrasonic mainframe, focusing system, coupling system, mechanical scanning devices etc. as in Fig. 3 [22]. 


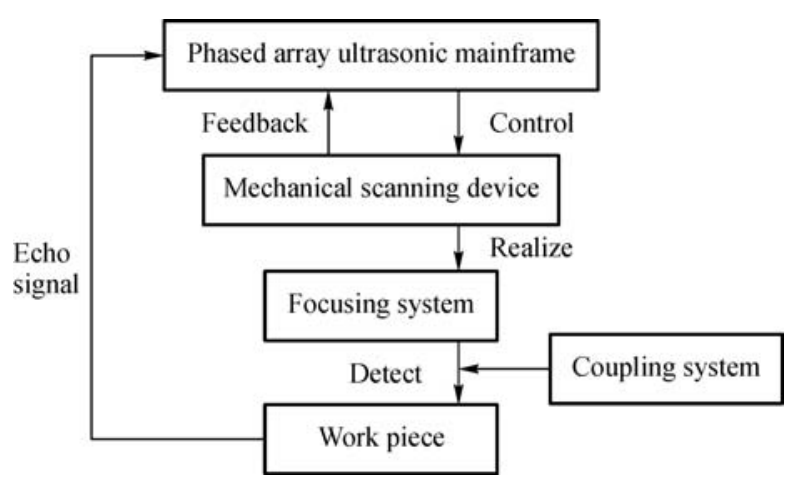

Fig. 3 Phased array ultrasonic system

In field ultrasonic testing of PE joints, several techniques have been innovated to improve inspection effectiveness. Reliable ultrasonic results can be achieved on the condition of proper transducer and optimized inspection parameters. Guo et al. [23] proposed a method of transducer design and parameter determination based on acoustic field simulation using CIVA software. With this method, all typical defects in EF joints can be imaged efficiently with good resolution and high signal-noise-ratio. Special ultrasonic system [24] was invented for BF joints applying coupling focusing inspection technique, including spherical probe immersed in couplant. Coupling focusing ultrasonic technique [25] was developed to reduce the sound energy loss at the interface between transducer and PE joints. The key of the technique is to invent a special couplant for PE. The couplant is a mixture of glycerinum, sodium silicate, water, defoamer, etc., which has almost the same sound velocity and acoustic impedance with PE.

$P E$ joints produced by different manufacturers may have grooves or textures on the surface. The irregular surface results in the expansion of a dead zone, deformation of detection figure, and reduction of sensitivity. A practical method [26] based on the coupling focusing ultrasonic technique was proposed to effectively reduce the unfavourable influence caused by irregular surface. PE joints generally have large fusion regions in circumferential direction, and volumetric inspection can be time-consuming. Automatic circumferential scanning devices for PE joint [27] were invented to enhance the inspection efficiency. Consequently, the ultrasonic images of the whole fusion region were reconstructed and displayed in real-time with the process of thorough scanning of PE joints.

\subsection{Defect recognition and safety assessment}

Considering that different types of defects have different failure modes and effects on pipeline safety, recognizing the type of detected flaw is crucial for further evaluation [28]. To improve defect recognition accuracy and realize in-service inspection, automatic defect recognition technique was developed [29]. Xie et al. [30] applied the principle of spatial compound imaging to reduce the noise in ultrasonic image, and consequently improved the image quality and defect detection ability. Rostami and Razavi [31] proposed a combined algorithm through various image processing and mathematical morphology to improve the raw ultrasonic images for observation and accurate analysis. Nevertheless, both the methods failed to differentiate defects from each other, and defect recognition still depends on experienced technicians. Huang et al. [32] summed the pixel grey-values in B-scan images of EF joint horizontally, normalized the value to one-dimensional signal, and analysed the signal by wavelet transform to facilitate automatic cold welding defect detection. Long et al. [33] conducted principal component analysis and regression analysis on eight features extracted from signal wave of voids and established the relation between the features and defect information. TWI [34] developed an automatic defect recognition (ADR) software for EF weld inspection, including three main steps in the algorithm: Detection of heating wire zone, determination of wire lines, and determination of defect maps. Based on the ADR software, defect indication can be completed, but defect classification and quantification required further analysis by technicians. Hou et al. [21] proposed a defect recognition method for $\mathrm{EF}$ joints based on pattern recognition principle, mainly including feature extraction and selection, defect classification, and defect quantification, as shown in Fig. 4. A series of software $[35,36]$ were further developed to perform automatic weld defect identification for phased array ultrasonic inspection, and the accuracy rate of single defect recognition reached $90 \%$.

Typical identification results are shown in Fig. 4. The left figures are digitalized ultrasonic images, and the right ones are B-scan image segmentations. Figure 4(a) is the image of normal welding without defect. Metal wires, void, poor fusion interface, and the eigen-line are marked with " + ", " $\triangle$ ", " $\diamond$ " and "blue line" as shown, respectively. Figure 4 shows that heating wires are recognized accurately, and all types of defects are successfully identified [21].

Safety assessment is necessary to estimate defect harmfulness and take measures after the defect recognition. A database system [37] on defect types, mechanical characteristics, and corresponding ultrasonic images of $\mathrm{PE}$ joints (EF and BF) was developed based on Visual Basic and SQL Server to provide substantial support for qualification of $\mathrm{PE}$ joints. Based on previous achievements of EF joints, Chinese national standard GB/T 29460-2012 specifies the characterization of all typical defects and stipulates the safety acceptance criteria, as shown in Table 1. Meanwhile, TWI [38] has developed a tensile test using a waisted specimen and a whole pipe tensile creep rupture test to determine flaw acceptance criteria for BF joints. However, achievements were only limited to planar lack of fusion flaws, and other critical flaws of BF joints were not considered. 

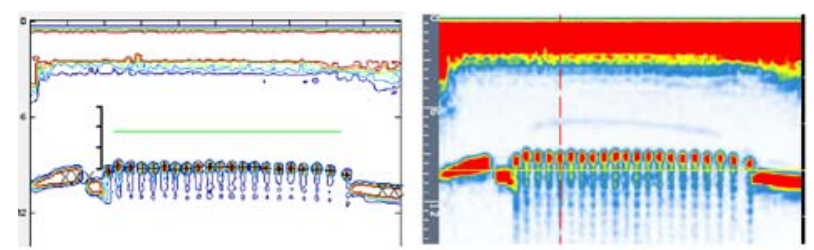

(a)
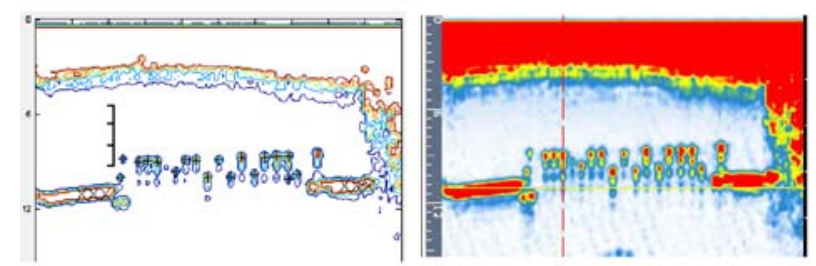

(b)

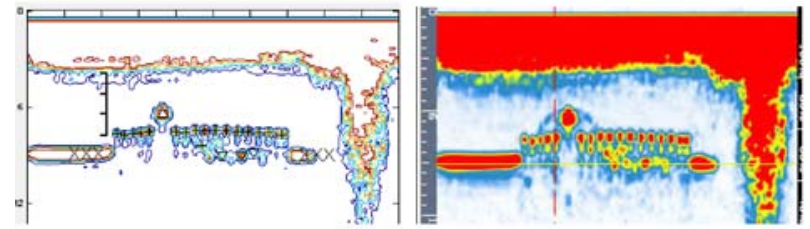

(c)
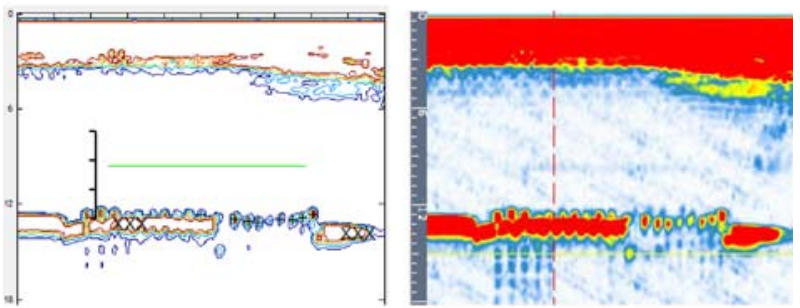

(d)
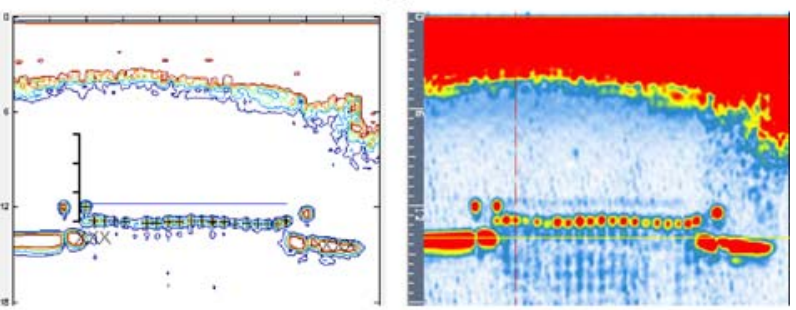

(e)

Fig. 4 Defect identification based on pattern recognition [21]. (a) Recognition of normal welding without defect; (b) recognition of metal wire dislocation; (c) recognition of voids; (d) recognition of poor fusion interface; (e) recognition of cold welding

\section{Ultrasonic inspection technique for PE pipe of large size}

General PAUT system can effectively inspect PE joints of DN40-400 for gas and water transportation covered by GB/T 29460-2012. However, for PE pipe of large size used in NPP, current technology was unable to adequately detect defects. A similar problem was also encountered for steel piping when the existing ultrasonic testing technique was applied to inspect thick-walled weld bead (more than 50 $\mathrm{mm}$ in thickness) [39-41]. The coarse grains in the welding region of the steel pipe lead to big noise, high sound attenuation, and deviation of sound path. These effects accumulate to a high level when ultrasound propagates in thick weld bead and consequently contribute to unreliable testing results. Therefore, process improvements $[42,43]$ for steel piping were conducted to raise the signal-to-noiseratio (SNR), and advanced ultrasonic techniques $[44,45]$ were innovated to extend the testing limit. Inspired by the ideas for steel piping ultrasonic testing inspection, studies on acoustic properties of PE were summarized as follows.

\subsection{Acoustic properties of PE}

Acoustic properties of PE were investigated based on molecule physics. Ultrasonic measurements, covering the frequency range of $5 \mathrm{MHz}$ to $1 \mathrm{GHz}$, were reported on linear and branched PE. The investigations [46] show that variations in attenuation are attributed to $\beta$ and $\gamma$ molecular relaxations, to spherulite-amorphous thermoelastic heat flow, and to spherulite-phonon scattering. Chain branching, annealing, and drawing all affect the crystallite morphology and thence the acoustic propagation parameters.

Meanwhile, relations between mechanical properties of $\mathrm{PE}$ and its acoustic properties were studied. Ultrasonic immersion method has been applied for the rapid and accurate measurement of acoustic attenuation and longitudinal sound velocity in PE. A study [47] on the highfrequency ultrasonic attenuation showed that an increase of the attenuation in PE was ascribed to scattering by domains of different modulus (crystallites). Furthermore, a systematic study [48] on PE samples of different grades showed that the velocity at $23{ }^{\circ} \mathrm{C}$ was proportional to density in the range of 0.92 to $0.97 \mathrm{~g} / \mathrm{cm}^{3}$. Other research [49] also supported that for a variety of low-density and high-density PE samples, sound velocity can be calculated by a function of the density and the crystallinity.

Several experimental investigations [50-54] were undertaken to study the velocity and attenuation of ultrasonic longitudinal waves in PE and their dependences on frequency. PE samples of different thickness in the range of 2.5 to $20.0 \mathrm{~mm}$ were tested using ultrasonic transducers with the central frequencies of 1,3 and $5 \mathrm{MHz}$. Though specific values of the ultrasonic parameters were not consistent from different experiments due to different PE grades used, all research concluded that the attenuation of longitudinal wave possessed almost linear dependence on frequency at $1-10 \mathrm{MHz}$, and the velocity also possessed strong dependence on frequency.

Therefore, unlike traditional metallic piping materials, $\mathrm{PE}$ is viscoelastic and its mechanical response at high frequency differs significantly from that at low frequency. For pulse propagation in such a medium [47], the strength of disturbance will significantly decrease with distance travelled, and different frequency components can travel at different velocities tending to distort the waveform, namely acoustic dispersion. Effects of acoustic attenuation 
Table 1 Defect quantification and safety assessment [10]

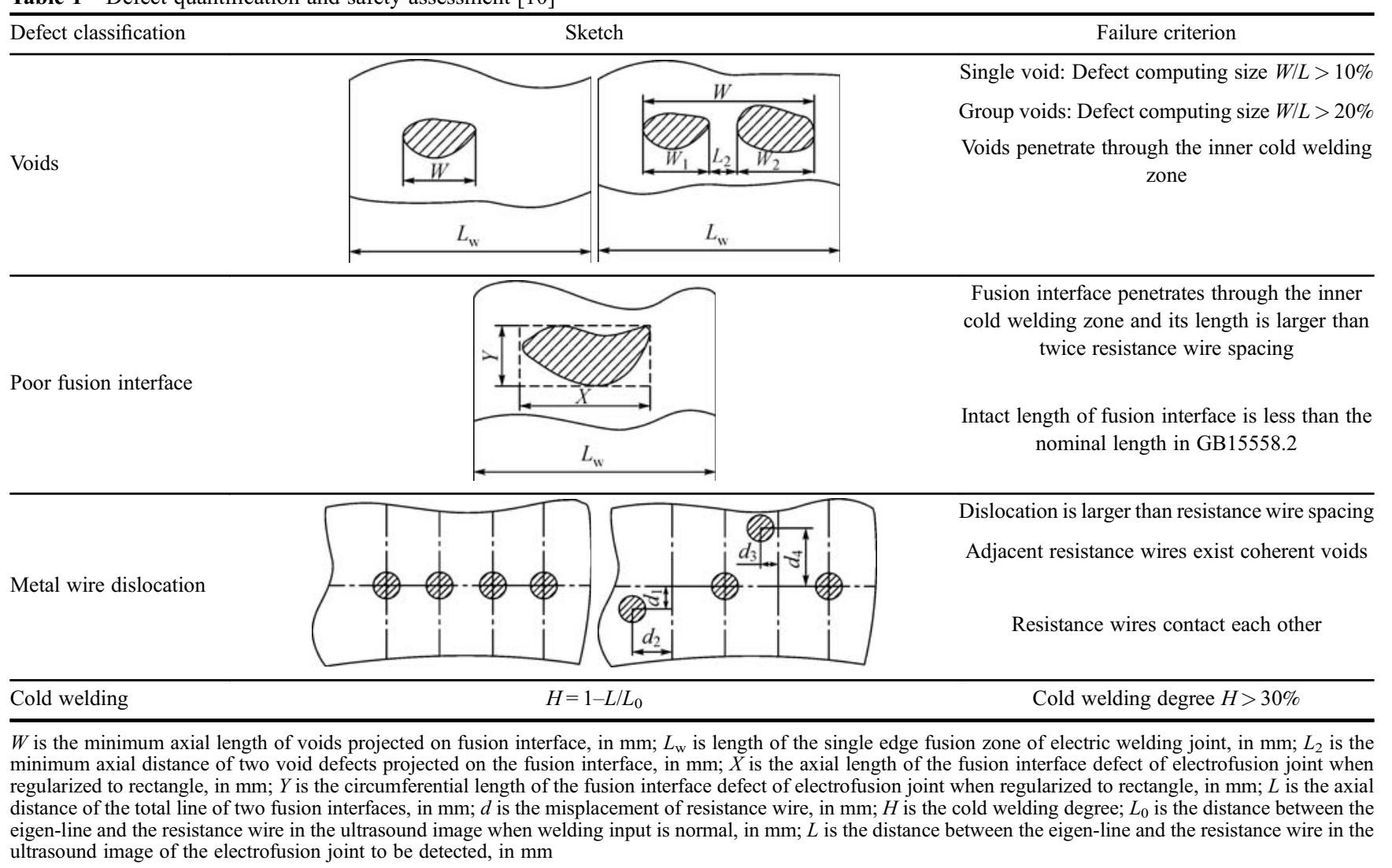

and acoustic dispersion on sound pressure distribution in the field accumulate with depth. Neglection of acoustic properties of PE was thought to be the main gap between the existing ultrasonic inspection techniques and that for PE joints of large size in NPP.

\subsection{Acoustic field model of PE}

Current ultrasonic inspection techniques are all based on ideal wave equation assuming tested materials of linear elasticity have constant sound velocity [55], as shown in Eq. (1). Under ideal conditions, the spatial resolution depends largely on the similarity of signals in shape and the precision of simple linear time delays determined by a constant sound speed [56]. Nevertheless, the attenuation and the dispersion of PE distort the acoustic waveform during the propagation through PE sample, therefore the ultrasonic spectrum cannot be converged. Hence, process of acoustic absorption of ultrasound in PE needs to be investigated quantitatively to broaden the application of the current ultrasonic inspecting technology.

$$
\frac{\partial^{2} P}{\partial z^{2}}-\frac{1}{c_{0}^{2}} \frac{\partial^{2} P}{\partial t^{2}}=0
$$

where $P$ is sound pressure (in $\mathrm{MPa}$ ), $z$ is propagation distance (in $\mathrm{mm}$ ), $t$ is propagation time (in $\mu \mathrm{s}$ ), and $c_{0}$ is sound velocity (in $\mathrm{mm} / \mu \mathrm{s}$ ).

The attenuation of acoustic waves propagating in a wide variety of lossy media obeys a power dependence on frequency of the general slowly varying form [54],

$$
\alpha(\omega)=\alpha_{0}|\omega|^{y}
$$

where $\omega$ is angular frequency, and $\alpha_{0}$ and $y$ are real nonnegative constants. For PE, the attenuation parameter $\alpha$ has a linear relation with frequency $\omega$, i.e., $y=1$.

Wave losses in acoustics are most often described either by relaxation phenomena given by equations similar to the form of the thermoviscous wave equation or its low frequency approximation. The thermoviscous wave equation [57] describes attenuation as a combined classical absorption that includes both viscous and thermal conduction losses but neglects acoustic dispersion and can only describe material corresponding to $y=2$. To deal with other materials, several methods [58,59] have been proposed including a superposition of relaxation frequencies in medical ultrasound and in geophysics, and a modification of the relaxation constant based on thermodynamic principles. Though the modified thermoviscous wave equation exhibits specific physical significance and is thought to be able to develop linear and nonlinear 
equations for most materials, the approach is restricted to complicated mathematical forms. Kramers-Kronig (K-K) relations [60] indicate that the real and imaginary components of the complex wavenumber are Hilbert transforms of each other, and thus K-K relations can be used in conjunction with the dispersion relation to obtain expressions linking the phase velocity to the attenuation coefficient. Nevertheless, the original K-K relation expressions are derived with the limitation of $0<y<1$. The use of a local K-K relation [61] was suggested for general case, but no method of converting these frequency relations to the time domain was given. Tavakkoli et al. [62] derived a relation between arbitrary absorption and dispersion directly in time domain based on the use of second-order operator splitting algorithm, but the relation was unable to describe sound reflection and diffraction.

Comparing dispersion relations based on the approximate thermoviscous acoustic wave equation and electromagnetic wave equation for a conducting medium, Szabo [63] proposed a causal time-domain wave equations for lossy media (for instance PE) of the power law type $(y=1)$ as shown in Eq. (3). Through experimental investigations [64], the equation proved effective in frequency domain to predict sound attenuation and phase velocity of PE at different frequencies and can be applied to study the acoustic field for ultrasonic inspection of PE joint of large size. The indirect implementation of Szabo's model is restricted by viable numerical solution of the convolutional integral, which for an accurate calculation, requires the complete time history of the acoustic field quantities. Recently, Ginter et al. [65] proposed an approach by transforming the original time-domain equations into complex discrete-time-frequency domain and then transforming it back into discrete time operators. This approach has difficulties in making a compromise between a sufficient approximation of the dispersion effects and the numerical efforts. Norton and Novarini [66] used a similar approach, but the approach cannot be used for twodimensional or three-dimensional problems because of the enormous computational cost. Currently, acoustic field distribution of PE cannot be solved directly in time domain.

$$
\frac{\partial^{2} P}{\partial z^{2}}-\frac{1}{c_{0}^{2}} \frac{\partial^{2} P}{\partial t^{2}}-\frac{8 \alpha_{0}}{\pi c_{0}} \int_{0}^{t} \frac{P\left(t-t^{\prime}\right)}{t^{\prime 3}} \mathrm{~d} t^{\prime}=0,
$$

where $\alpha_{0}$ is attenuation coefficient (in $\mathrm{Np} /(\mathrm{mm} \cdot 2 \pi \cdot \mathrm{MHz})$ ), and $c_{0}$ is reference sound velocity (normally corresponding to center frequency, in $\mathrm{mm} / \mu \mathrm{s}$ ).

\subsection{Field inspection trials of PE pipe of large size}

Ultrasound waveform changes when propagating in PE. On one hand, sound wave has an obvious frequency downshift with the increase of depth. Actual time delay of target signal transmitting in PE differs from that of pulse transmitting in non-dispersive medium, and the differentiation enlarges with depth and for high frequency [64]. Improvements were made to minimize the influence of acoustic attenuation and dispersion in PE on the inspection effectiveness under existing technical conditions.

For EF joint of large size $(710 \mathrm{~mm}$ in outer diameter (OD), $40 \mathrm{~mm}$ in thickness), TWI [67] applied PAUT technique with low testing frequency $(5 \mathrm{MHz})$ to achieve sufficient propagation distance of the sound considering that PE is a highly attenuating material. The result in Fig. 5(a) shows that only part of the metal wires can be recognized, and the SNR needs further improving. For BF joint of large size (630 $\mathrm{mm}$ in OD, $60 \mathrm{~mm}$ in thickness), TWI [68] applied a developed PAUT system utilising membrane water wedges for low attenuation. The result in Fig. 5(b) indicates that defects at a depth of $21 \mathrm{~mm}(42 \mathrm{~mm}$ in sound path) in the BF joint can be inspected with the technique, but the image qualification was less satisfactory with low SNR. Zheng et al. [69] innovated the probe for EF joint (762 $\mathrm{mm}$ in OD, $80 \mathrm{~mm}$ in thickness) used in NPP and optimized principal testing parameters by comprehensively considering the testing effectiveness including sensitivity, penetration, SNR, resolution, and accuracy. Typical defects were found in field inspection. As shown in Fig. 5(c), the maximum wire displacement was approximately $3.3 \mathrm{~mm}$. However, the metal wires in the ultrasonic image displayed has a width of $5 \mathrm{~mm}$, compared with the actual diameter of $3 \mathrm{~mm}$. Therefore, the inspection techniques should be further developed in the testing resolution enhancement for accurate defect quantification and safety assessment.

\section{Prospects of ultrasonic inspection technology for PE pipe in NPP}

In the first section, gaps in the code case of N755 that pertain to the volumetric inspection are identified. Identifying gaps is an important step to develop the standards needed. Based on the previous analysis, the principal technical obstacle of innovating reliable NDE technology for PE pipe of large size was the neglection of acoustic attenuation and dispersion in PE when applying the existing ultrasonic inspection technology. To complete the NDE section of standards or cases for PE pipe used in NPP, the following technical issues should be solved beforehand:

1) Research on phased array focal field

Phased array focal field research provides the theoretical basis of investigating actual acoustic field characteristics, recovering the wave form and further developing an effective ultrasonic phased array system. For large diameter PE pipe joint in NPP, accumulating effects of acoustic attenuation and dispersion on ultrasound wave propagating in PE should be taken into account. Consequently, time-domain field distribution of plane wave 


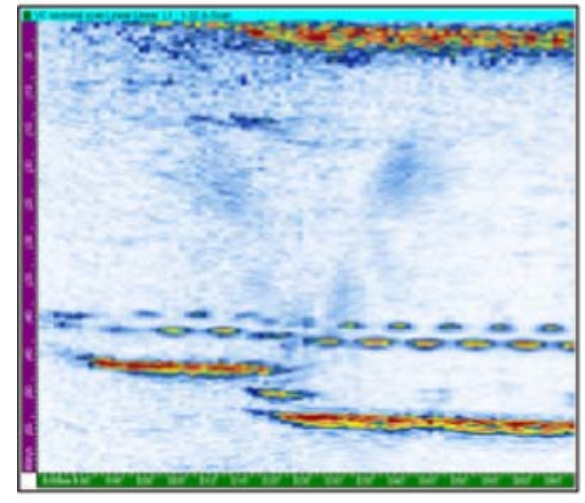

(a)

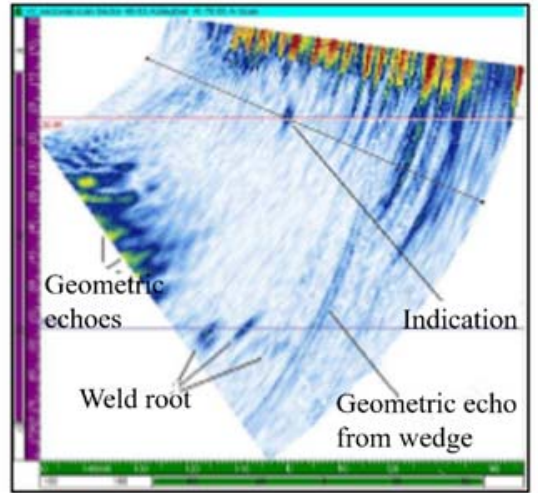

(b)

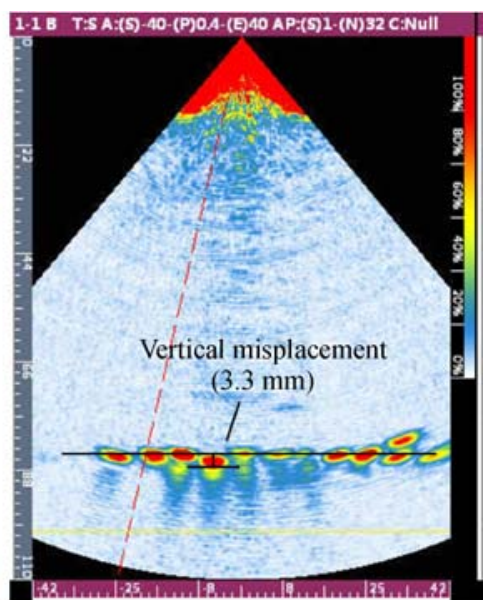

(c)

Fig. 5 Ultrasonic image of PE joint of large size. (a) EF joint of D710 [67]; (b) BF joint of D630 [68]; (c) EF joint of D762 [69]

based on the causal theory needs to be first solved, and then elementary spherical wave field of phased array ultrasound can be obtained. Considering the distortion of pulse waveform, the actual time delay of transducer should be calculated to realize the expected focal region in large diameter PE joint. Furthermore, the phased array focal field can be determined through superposition of waves driven by all excited elements. Focal field of phased array ultrasound is key to defect detection. For high imaging precision, scattering principle of all typical defects and corresponding scattered ultrasound field distribution should be also clarified.

2) Development of special ultrasonic inspection system

Phased array ultrasonic inspection system should be innovated specially for PE joint of large size used in NPP. Delay time algorithm, imaging principle, and gain margin should be programmed to consider the acoustic properties of PE. Meanwhile, all related devices such as probe and filtering circuit need to be redesigned correspondingly to satisfy new inspection requirements. In comparison with EF joint, emitting ultrasound wave generates both longitudinal and transversal wave in BF joint after passing through the testing wedge. Therefore, the influence of attenuation and dispersion of PE on the transversal wave should be also studied. On the basis of acoustic properties of composite sound field, the optimal testing parameters for PE joint of large size should be investigated with inspection effectiveness index comprehensively considered. Lastly, PE joint in NPP usually has complicated operation surroundings and complex testing surface, so appropriate testing procedures and assisting techniques can increase efficiency and ensure reliability.

3) Qualification of defected PE joint

Phased array ultrasonic inspection technique is available to measure the characteristics of volumetric defects in PE joints used in NPP. However, additional research is required to specify the critical dimensions of a specific defect associated with pressure and temperature that the joint will experience during its life span. The critical dimensions of inspected defects should be characterized and quantified and reflect the risk of failure for the joint in both short-term and long-term strength.

\section{Conclusions}

PE pipe exhibits excellent advantages over traditional metal pipe for some cooling applications in NPP. Lack of reliable NDE method is the main barrier for ensuring the long-term safety of HDPE pipe in NPP. This paper reviewed current development in nondestructive test technology for PE pipe, and the main conclusions are drawn as follows:

1) Phased array ultrasonic inspection technique exhibits great advantages for the detection of all typical defects in both EF joints and BF joints, especially for cold welding. Based on the technique, a number of ultrasonic testing techniques have been developed for PE joint (DN40-400), including special ultrasonic inspection system, automatic defect recognition, and safety assessment.

2) For long distance sound propagation in viscoelastic medium like PE, acoustic attenuation and dispersion should be taken into consideration to attend to the existing ultrasonic inspection technique to large diameter PE joints. By minimizing the influence of acoustic attenuation and dispersion, PAUT has been successfully used to detect defects in EF joint (762 $\mathrm{mm}$ in OD, $80 \mathrm{~mm}$ in thickness), but the resolution needs further improvement.

Research on the safety assessment of PE pipe joint containing defects is suggested in the instructions of the ultrasonic inspection, and acceptance criteria can be specified in related standards for PE pipe in NPP.

Acknowledgements The authors gratefully acknowledge the financial 
support from the National Natural Science Foundation of China (Grant No. 51575480) and the Fundamental Research Funds for the Central Universities (Grant No. 2017FZA4012).

Open Access This article is distributed under the terms of the Creative Commons Attribution 4.0 International License (http://creativecommons.org/ licenses/by/4.0/), which permits unrestricted use, distribution, and reproduction in any medium, provided the appropriate credit is given to the original author(s) and the source, and a link is provided to the Creative Commons license, indicating if changes were made.

\section{References}

1. Zhang D. Development of nuclear power in the world and its enlightenment to China. Energy Technology and Economics, 2010, 22(12): 5-10 (in Chinese)

2. Shu G, Xue F, Ti W, et al. Flow accelerated corrosion and aging management in nuclear power plants. Corrosion and Protection, 2006, 27(2): 72-76 (in Chinese)

3. Crawford S L, Steven R, Cinson A D, et al. Assessment of NDE methods to detect lack of fusion in HDPE butt fusion joints. In: Proceedings of ASME 2011 Pressure Vessels and Piping Conference. Baltimore: ASME, 2011, 1: 343-349

4. Installations M. PPI Handbook of Polyethylene Piping. 2007. Retrieved from http://plasticpipe.org/publications/pe-handbook. html

5. Abel S L, Brandes M D, Corley L J, et al. Use of HDPE piping in the Callaway nuclear plant essential service water system. In: Proceedings of ASME 2009 Pressure Vessels and Piping Conference. Prague, 2009, 1285-1293

6. Golliet M G. Update on code case N-755 revision class 3 polyethylene piping. In: Proceedings of International Conference on Nuclear Engineering. Brussels, 2009, 219-223

7. American Society of Mechanical Engineers. ASME Code Case N755-2, Use of Polyethylene (PE) Class 3 Plastic Pipe, Section III, Division 1. 2015

8. Crawford S L, Cumblidge S E, Doctor S R, et al. Technical letter report-Preliminary assessment of NDE methods on inspection of HDPE butt fusion piping joints for lack of fusion. In: Proceedings of ASME 2010 Pressure Vessels and Piping Division/K-PVP Conference. Richland: Pacific Northwest National Laboratory, 2009, 1039-1045

9. Krishnaswamy P, Focht E M, Shim D J, et al. Use of polyethylene (PE) pipe in safety-related, Class 3, service-water piping. In: Proceedings of 16th International Conference on Nuclear Engineering. Orlando: ASME, 2008, 1019-1023

10. General Administration of Quality Supervision, Inspection and Quarantine of the People's Republic of China, Standardization Administration of China. Safety assessment for electrofusion joint of polyethylene pipes containing defects. GB/T 29460-2012, 2012

11. General Administration of Quality Supervision, Inspection and Quarantine of the People's Republic of China, Standardization Administration of China. Ultrasonic testing for electrofusion joint of polyethylene pipe. GB/T 29461-2012, 2012

12. Shi J, Zheng J, Guo W, et al. Defects classification and failure modes of electrofusion joint for connecting polyethylene pipes.
Journal of Applied Polymer Science, 2012, 124(5): 4070-4080

13. Guo W. Ultrasonic testing technique researcher and equipment fabrication of polyethylene butt fusion joint. Dissertation for the Doctoral Degree. Hangzhou: Zhejiang University, 2014 (in Chinese)

14. Shi J, Zheng J, Guo W, et al. Safety assessment of cold welding defect in electro-fusion joint of polyethylene pipe. In: Proceedings of ASME 2012 Pressure Vessels and Piping Conference. Toronto: ASME, 2012, 189-196

15. Nie X, Hou D, Zheng J, et al. Eigen-line in welded structures of thermoplastic polymers. Polymer Testing, 2017, 57: 209-218

16. Zhao H. Welding technology and evaluation method of polyethylene pipes. China Plastics, 2011, 7: 48-53 (in Chinese)

17. Han S, Xia F, Chen H. Ultrasonic inspection of butt fusion joint of polyethylene (PE) pipe for gas. China Special Equipment Safety, 2007, 9: 55-57 (in Chinese)

18. Qiu X, Zhong S, Zhu Z, et al. Infrared thermography simulation and experiments of polyethylene pipe defects. Mechanical \& Electrical Engineering Magazine, 2014, 31(12): 1513-1517 (in Chinese)

19. Wang S, Li J, Li T, et al. Fuzzy evaluation of ultrasonic testing effect of PE pipe weld defect. China Measurement \& Test, 2016, 42(8): 39-43 (in Chinese)

20. Spicer M, Troughton M, Hagglund F. Development and assessment of ultrasonic inspection system for polyethylene pipes. In: Proceedings of ASME 2013 Pressure Vessels and Piping Conference. Paris: ASME, 2013, 2536-2545

21. Hou D, Guo W, Zheng J. A method of automatic defect recognition for phased array ultrasonic inspection of polythene electro-fusion joints. In: Proceedings of ASME 2015 Pressure Vessels and Piping Conference. Boston, 2015, V5T-V9T

22. Bao X. Research on phased array ultrasonic testing system and its key technology. Dissertation for the Doctoral Degree. Beijing: Tsinghua University, 2003 (in Chinese)

23. Guo W, Shi J, Hou D. Phased array ultrasonic technology optimization for electro-fusion joint of polyethylene pipe. Nondestructive Testing, 2015, 37(8): 30-34 (in Chinese)

24. Ding S, Shi J, Zheng J, et al. China Patent, CN 101393170 B, 201009-22

25. Guo W, Hong S, Zhu L, et al. Experimental investigation on coupling focusing ultrasonic technique for inspection of polyethylene butt fusion joint. Nondestructive Testing, 2014, 8(10): e77442 (in Chinese)

26. Guo W, Zheng J, Ding S, et al. Analysis on the influence of the irregular surface of electro-fusion joint when tested by phased array ultrasonic technique. Pressure Vessel Technology, 2009, 26(2): 6-10 (in Chinese)

27. Zheng J, Guo W, Shi J, et al. China Patent, CN201310466694, 2016-03-16

28. Ravanbod H, Karimi F, Amindavar H. Flaw characterization in ultrasonic non-destructive testing method using exponential modeling. In: Proceedings of 2013 IEEE International Instrumentation and Measurement Technology Conference. Minneapolis: IEEE, 2013, 1676-1679

29. Ravanbod H, Jalali A. Configurable ultrasonic flaw classification of oil pipelines. Nondestructive Testing and Evaluation, 2008, 23(1): $43-55$

30. Xie C, Wang S, Zhang J, et al. Spatial compounding imaging in 
ultrasonic test for joint of polyethylene pipe. Nondestructive Testing, 2014, 36(11): 49-53 (in Chinese)

31. Rostami B, Razavi B. Application of digital image processing on nondestructive testing of fusion joints of polyethylene piping by ultrasonic technique. In: Proceedings of International Conference on Wireless Communications \& Signal Processing. Nanjing, 2011

32. Huang Y, Zhong S, Fu X, et al. Cold welding defect detection of electrofusion joints in polyethylene pipes based on wavelet analysis. Nondestructive Test, 2015, 39(1): 6-10 (in Chinese)

33. Long S, Yu R, Ma J. The extracting and analyzing on the features of ultrasonic testing signal getting from $\mathrm{PE}$ butt weld. Nondestructive Testing, 2009, 31(6): 442-444 (in Chinese)

34. Spicer M, Troughton M, Hagglund F. Development and assessment of ultrasonic inspection system for polyethylene pipes. In: Proceedings of ASME 2013 Pressure Vessels and Piping Conference. Paris: IEEE, 2013, 2536-2545

35. Zhejiang University. Automatic recognition software of polyolefin composite pipe welding joints for ultrasonic phased array flaw detection. V1.0 ed. 2014

36. Zhejiang University, Ningbo Aupul Pipe Technology Co Ltd. Automatic recognition software of polyolefin composite pipe welding joints for ultrasonic phased array flaw detection. V2.0 ed. 2016

37. Wang F, Li G, Shao H, et al. Development of ultrasonic detection database of polyethylene pipe joints. China Plastics, 2010, 6: 86-90 (in Chinese)

38. Troughton M, Khamsehnezhad A. Short-term and long-term mechanical testing to evaluate the effect of flaws in butt fusion joints in polyethylene pipes. In: Proceedings of ASME 2016 Pressure Vessels and Piping Conference. Vancouver: ASME, 2016, V06BT06A050

39. Shahjahan S, Aubry A, Rupin F, et al. Parametrical study of flaw detection in polycrystalline materials by reducing the multiple scattering contribution. AIP Conference Proceedings, 2013, 1511 (1): 675-682

40. Ramuhalli P, Good M S, Diaz A A, et al. Ultrasonic characterization of cast austenitic stainless-steel microstructure: Discrimination between equiaxed- and columnar-grain material-An interim study. Office of Scientific \& Technical Information Technical Reports PNNL-18912. 2009

41. Jenson F, Fortuna T, Doudet L. Modeling of ultrasonic propagation in a coarse grain structure. AIP Conference Proceedings, 2009, 1096 (1): 1201-1208

42. Guo W, Zheng J, Liu Z, et al. The experimental investigation of ultrasonic testing sensitivity for internal surface radial defects in cylindrical item with thick wall. Nondestructive Testing, 2012, 34 (2): 24-27 (in Chinese)

43. Han Z, Wang B, Yuan K, et al. Ultrasonic scanning imaging for austenitic thick-wall welds. Journal of Materials Engineering, 2012, 9(2): 62-65

44. Lu P. Research on ultrasonic detection technology of thick-wall parts in power station. Thesis for the Master's Degree. Baoding: North China Electric Power University, 2013 (in Chinese)

45. Xiao K, Wang Q, Hu D, et al. Simulation analysis of internal defects in thick-walled pipes with ultrasonic flexible array probes. Journal of Wuhan University of Technology (Transportation Science \&
Engineering), 2013, 37(2): 416-419 (in Chinese)

46. Felix M P. Attenuation and dispersion characteristics of various plastics in the frequency range 1-10 MHz. Journal of Composite Materials, 1974, 8(3): 275-287

47. Murata K, Li Y, Tanaka Y, et al. Measurement of acoustic attenuation and dispersion in low-density polyethylene by pulsed electro-acoustic method. IEEJ Transactions on Fundamentals and Materials, 1995, 115: 344-348

48. Adachi K, Harrison G, Lamb J, et al. High frequency ultrasonic studies of polyethylene. Polymer, 1981, 22(8): 1032-1039

49. Piché L. Ultrasonic velocity measurement for the determination of density in polyethylene. Polymer Engineering and Science, 1984, 24 (17): 1354-1358

50. Davidse P D, Waterman H I, Westerdijk J B. Sound velocity and Young's modulus in polyethylene. Journal of Polymer Science, 1962, 59(168): 389-400

51. Mažeika L, Šliteris R, Vladišauskas A. Measurement of velocity and attenuation for ultrasonic longitudinal waves in the polyethylene samples. Ultrasound, 2010, 65(4): 12-15

52. Kline R A. Measurement of attenuation and dispersion using an ultrasonic spectroscopy technique. Journal of the Acoustical Society of America, 1984, 76(2): 498-504

53. Raišutis R, Kažys R, Mažeika L. Application of the ultrasonic characterization methods for highly attenuating plastic materials. NDT \& E International, 2007, 40(4): 324-332

54. Farhat G. Diagnostic Ultrasound Imaging: Inside Out. Philadelphia: Lippincott-Raven, 2007

55. Du G. Acoustic Foundation. Shanghai: Shanghai Scientific and Technical Publishers, 1981 (in Chinese)

56. Shi K, Guo Y. Phased Array Ultrasonic Imaging and Testing. Beijing: Higher Education Press, 2010 (in Chinese)

57. Pierce A D, Smith P W. Acoustics: An introduction to its physical principles and applications. Physics Today, 1981, 34(12): 56-57

58. Nachman A I, Smith III J F, Waag R C. An equation for acoustic propagation in inhomogeneous media with relaxation losses. Journal of the Acoustical Society of America, 1990, 88(3): 15841595

59. Liu H, Anderson D, Kanamori H. Velocity dispersion due to anelasticity; implications for seismology and mantle composition. Geophysical Journal International, 1976, 47(1): 41-58

60. Kronig R D L. On the theory of dispersion of X-ray. Journal of the Optical Society of America, 1926, 12(6): 547-556

61. O’Donnell M, Jaynes E T, Miller J G. Kramers-Kronig relationship between ultrasonic attenuation and phase velocity. Journal of the Acoustical Society of America, 1981, 69(3): 696-701

62. Tavakkoli J, Cathignol D, Souchon R, et al. Modeling of pulsed finite-amplitude focused sound beams in time domain. Journal of the Acoustical Society of America, 1998, 104(4): 2061-2072

63. Szabo T L. Time domain wave equations for lossy media obeying a frequency power law. Journal of the Acoustical Society of America, 1994, 96(1): 491-500

64. Sheng X, Hou D, Zheng J. Investigation on acoustic propagation of ultrasound in polyethylene pipe used in nuclear power plant. In: Proceedings of ASME 2017 Pressure Vessels and Piping Conference. Waikoloa: IEEE, 2017, V03AT03A049

65. Ginter S, Liebler M, Steiger E, et al. Full-wave modeling of 
therapeutic ultrasound: Nonlinear ultrasound propagation in ideal fluids. Journal of the Acoustical Society of America, 2002, 111(5): 2049-2059

66. Norton G V, Novarini J C. Including dispersion and attenuation directly in the time domain for wave propagation in isotropic media. Journal of the Acoustical Society of America, 2003, 113(6): 30243031

67. Hagglund F, Spicer M, Troughton M. Development of phased array ultrasonic inspection techniques for testing welded joints in plastic
(PE) pipes. In: Proceedings of 18th World Conference on Nondestructive Testing. Durban, 2012

68. Hagglund F, Robson M, Troughton M J, et al. A novel phased array ultrasonic testing (PAUT) system for on-site inspection of welded joints in plastic pipes. In: Proceedings of National Seminar \& Exhibition on Non-Destructive Evaluation. Pune, 2014

69. Zheng J, Hou D, Guo W, et al. Ultrasonic inspection of electrofusion joints of large polyethylene pipes in nuclear power plants. Journal of Pressure Vessel Technology, 2016, 138(6): 060908 